\title{
¿Son los códigos éticos un instrumento de gestión de la responsabilidad social? Un estudio exploratorio en Andalucía
}

\author{
Mercedes Ruiz-Lozano, Araceli de los Ríos Berjillos, Salud Millán Lara \\ Universidad Loyola Andalucia (Spain) \\ mruiz@uloyola.es,arios@uloyola.es,smillan@uloyola.es
}

Received July, 2015

Accepted January, 2016

\section{Resumen}

Objeto: La mayor preocupación por la responsabilidad social y/o la ética empresarial debe tener su reflejo en los sistemas de control de las organizaciones. Por ello nos preocupa analizar si se han introducido los códigos éticos como instrumento de gestión de la responsabilidad social, para construir organizaciones sostenibles basadas en valores.

Diseño/metodología/enfoque: Elaboración de una encuesta fundamentada en la revisión bibliográfica sobre tipos de códigos y motivaciones. Se ha realizado un análisis descriptivo de la información obtenida de las empresas con sede en Andalucía objeto de estudio. Además se ha aplicado un test de diferencia de medias y un análisis de la varianza para contrastar las hipótesis planteadas.

Aportaciones y resultados: Se ha identificado una cierta tendencia a utilizar los códigos éticos por parte de las empresas andaluzas, influida por el tamaño empresarial. Además se confirma la incidencia del entorno en su desarrollo.

Limitaciones: El estudio tiene carácter exploratorio en el ámbito andaluz por las restricciones de las bases de datos y del tamaño de la muestra. 
Implicaciones prácticas: Los resultados permiten un acercamiento a la realidad empresarial andaluza y a los sistemas de gestión relacionados con la responsabilidad social y la ética empresarial.

Implicaciones sociales: Se ha puesto de manifiesto la necesidad de formación en este ámbito y que desde el sector público se adopten medidas de fomento de la ética y de las responsabilidad social empresarial distinguiendo entre las necesidades que puedan tener las micro y pequeñas empresas de las que puedan tener las medianas o las grandes empresas.

Originalidad / Valor añadido: Este estudio contribuye a generar conocimiento sobre la importancia de integrar en los sistemas de control de gestión instrumentos que favorezcan una gestión responsable y sostenible basada en valores y sobre cuáles han sido las motivaciones que están influyendo tanto a favor como en contra de esta actuación. Motivaciones que habría que tener en cuenta por parte de los organismos decisores e influyentes en el desarrollo empresarial.

Palabras clave: Códigos éticos, Responsabilidad social, Sistemas de gestión, Andalucía, Pymes

\section{Códigos JEL: M1}

Title: Are the ethical codes a management tool of social responsibility? An exploratory study in Andalusia

\section{Abstract}

Purpose: The growing concern for social responsibility and/or business ethics must be reflected in the control systems of organizations. Therefore we are concerned to analyze whether the ethical codes have been introduced as a management tool of social responsibility to build sustainable value-based organizations.

Design/methodology: Development of a survey based on the literature review of code types and motivations. We performed a descriptive analysis of the information obtained from companies based in Andalusia under study. Furthermore, it has been implemented a test of mean difference and an analysis of variance to contrast the hypotheses.

Findings: The study supports a tendency to use the ethical codes by Andalusian companies analyzed, influenced by firm size. Similarly, the incidence of immediate environment is confirmed. 
Research limitations/implications: The study is exploratory in the Andalusian area due to restrictions databases and sample size.

Practical implications: The results allow an approach to the Andalusian business reality and to the management systems related with social responsibility and business ethics.

Social implications: It has highlighted the need for training in this area and that measures to promote ethics and corporate social responsibility are taken from the public sector, distinguishing between needs that may have micro and small enterprises from medium or large companies.

Originality/value: This study contributes to knowledge about the importance of integrating instruments that promote responsible and sustainable management based on values in management control systems and about what were the motivations that are influencing both, for and against, in this action. Motivations that should be taken into account by decisionmakers and influential in business development agencies.

Keywords: Ethical codes, Social responsibility, Management systems, Andalusia, SMEs

Jel Codes: M1

\section{Introducción}

Las múltiples noticias sobre corrupción de los últimos años han priorizado, en el ámbito empresarial, todo lo relacionado con la ética y la responsabilidad social (RS) como elementos vertebradores de la gestión de las organizaciones.

Un mundo complejo, presidido por la globalización, las nuevas tecnologías, la presión del tiempo, la innovación continua y un entorno cambiante, requiere la consideración de criterios éticos para orientar el comportamiento humano, tanto en las decisiones personales como en las de las organizaciones, porque detrás de éstas es el ser humano el que está presente en todas ellas y es sobre el que recaen las consecuencias. En este sentido, Melé (1999) afirma que la ética empresarial tiene que orientar cualquier acto, porque la ética es algo consubstancial a la acción, y como la acción empresarial incide en las personas involucradas en ella, toda acción tiene un componente ético. 
Investigadores, entre los que se encuentran Russo y Perrini (2010) consideran que las estrategias o políticas de responsabilidad social de las grandes y pequeñas empresas son una respuesta a la teoría de los stakeholders que contribuye al desarrollo crítico de la ética empresarial.

Diferentes estudios han corroborado un círculo virtuoso derivado del liderazgo ético en las organizaciones. Un comportamiento ético supone mejores resultados operativos y una mayor integridad en la organización, lo que a su vez generalmente se traduce en un mayor nivel de transparencia con la consecuente valoración de todos los grupos de interés (Toro, 2006).

Pero para conseguir el liderazgo ético hay que integrar en la gestión de la organización los valores que guían ese comportamiento. Uno de los instrumentos de gestión recomendados para dar una respuesta a esa cuestión son los códigos éticos o códigos de conducta (Kaptein, 2004). Pese a las dudas que suscita su eficacia, hay un acuerdo bastante generalizado de que la institucionalización y comunicación de un código ético es el primer paso para la mejora del comportamiento ético de una organización en relación con sus diferentes stakeholders, y que es uno de los instrumentos que es utilizado tanto por las grandes empresas como por las Pymes para gestionar su RS o su capital social (Russo \& Perrini, 2010).

La investigación sobre códigos éticos se sustenta en las principales teorías sociales, en concreto, la teoría de los stakeholders y la teoría de la legitimidad. La teoría de los stakeholders sostiene que las empresas son responsables ante cualquier agente o grupo de interés que sea afectado por las acciones de la organización. Desde esta perspectiva, los códigos éticos son un instrumento al alcance las empresas para guiar la respuesta a las expectativas de sus grupos de interés, y orientar el diálogo con ellos. Para la teoría de la legitimidad, ésta se deriva de la congruencia entre el sistema de valores de una organización y el de la sociedad de la que forma parte. Desde este enfoque, la incorporación de códigos éticos es un buen mecanismo para legitimar el comportamiento de la empresa, teniendo en cuenta que la empresa no sólo debe hacer lo que se espera de ella, sino que también necesita justificar ante la sociedad la orientación de sus actividades y cambios internos (García, Rodríguez \& Gallego, 2008).

Diferentes motivaciones han llevado a las grandes empresas a implementar códigos éticos, entre las que destacan la protección o mejora de la reputación, la atención a las expectativas de los stakeholders clave, la formalización y comunicación de compromisos, el suscitar y mantener la confianza, y la atención a los requerimientos legales, entre otros (Bondy, Matten \& Moon, 2008).

Esa mayor preocupación por la responsabilidad social y/o la ética debe tener su reflejo en los sistemas de control de la organización. En los últimos años este interés se ha visto reflejado en la investigación realizada acerca de la integración de los códigos en grandes empresas y/o multinacionales (Wood, 
Svensson, Singh, Carasco \& Callaghan, 2004; Sethi, 2003; Singh, 2006; Rodríguez-Domínguez, GarcíaSánchez \& Gallego-Álvarez 2009) pero son pocos los estudios que han analizado el comportamiento de las Pymes y, de forma más concreta, los instrumentos de gestión de la responsabilidad social que utilizan (Turyakira, Venter \& Smit, 2012).

En España la inquietud relacionada con el comportamiento ético en las empresas se observa en los distintos desarrollos de códigos de buen gobierno, Código Unificado de Buen Gobierno de las Sociedades Cotizadas, y en la reciente Ley 31/2014, de 3 de diciembre, por la que se modifica la Ley de Sociedades de Capital para la mejora del gobierno corporativo. Expresamente la Ley 31/2014 menciona entre sus objetivos mejorar el control interno y la responsabilidad corporativa de las empresas españolas. Otra manifestación es la preocupación surgida por la incorporación de códigos éticos como parte de los procesos de prevención y control de los programas de cumplimiento normativo derivados de la reforma del Código Penal

Por otra parte, el tejido empresarial andaluz se caracteriza, al igual que en el resto de España, por la presencia de las Pymes. El 99.8\% de las empresas en España tienen menos de 50 trabajadores y de ellas el 15\% son andaluzas. En Andalucía representaron un 99.9\% del total de empresas, según datos del INE correspondientes a 2014; una décima más que la media nacional. Además, en su mayoría, el 96.5\%, son empresas de menos de 9 trabajadores, esto es, microempresas. Este porcentaje también es superior a la media nacional, donde éstas representan el 95.8\% del total de empresas.

La menor capitalización de las Pymes, sus tradicionales dificultades para acceder al crédito, a la tecnología o para atraer el talento, son algunas de las razones por las que, precisamente, muchas de ellas están viendo en la responsabilidad social una estrategia para competir acorde con sus valores, adaptada a sus recursos y fuente de innovación.

Esta investigación se realiza como parte de una línea de investigación de la Universidad Loyola Andalucía, acerca de la integración de la Responsabilidad Social en la gestión de las organizaciones y de su diálogo con los grupos de interés. Por lo que como inicio de su investigación, en relación con la incorporación de los códigos éticos como instrumento de gestión de la responsabilidad social en las empresas, se ha limitado el ámbito de estudio a las empresas andaluzas con sede en Andalucía colaboradoras con la Universidad.

Por ello, son objeto específico de esta investigación conocer si el código ético es un instrumento incorporado en los sistemas de gestión de las empresas con sede principal en Andalucía y cuáles son realmente sus motivaciones. Un análisis atendiendo a los diferentes tamaños permitirá detectar su 
influencia en la decisión de desarrollar un código, en el tipo de código y en sus motivaciones, lo que a su vez conducirá la propuesta de actuaciones que se incluirán en las conclusiones.

El presente trabajo comienza con una breve referencia a los códigos éticos como instrumento de gestión de la responsabilidad social, de los valores que conforman la política de la empresa con sus stakeholders. A continuación se describe la metodología utilizada en esta investigación, que junto con la presentación de los resultados obtenidos nos llevarán a terminar con las conclusiones derivadas del estudio.

\section{Los códigos éticos como instrumento de gestión de la responsabilidad social}

Los códigos éticos son documentos formales que expresan, en un lenguaje fácilmente comprensible, los valores esenciales de una organización y las normas éticas y principios a los que deben adherirse los empleados de una empresa (Adams, Tasachian \& Stone, 2001; Valentine \& Barnett, 2003). Esto es, el conjunto de normas morales que guían el comportamiento de la organización y que le dan identidad. No se trata de recoger en un documento, normas y regulaciones específicas, sino de explicitar los valores de la organización para que orienten sus acciones concretas. Así entendidos, los códigos no sólo no encorsetan a la organización sino que la dotan de flexibilidad, de innovación y creatividad y de responsabilidad, con el único límite del respeto a los valores orientadores de la actividad (Kaptein, 2004).

Este enfoque ha dado lugar a una evolución del código ético, que es identificado por las empresas como código de conducta, política ética, declaración de buenas prácticas, principios profesionales o principios de responsabilidad social, entre otros, (Schwartz, 2001), para guiar en el desarrollo de su actividad las relaciones con los distintos stakeholders.

El desarrollo de los códigos ha derivado en una diversidad de tipos en función de la inclusión de aspectos o contenidos, de su estructura y de su utilidad (Jenkins, 2001; Rezaee, Elmore \& Szendi, 2001; Preuss, 2009; Ruiz-Lozano \& Araque-Padilla, 2013).

Pero a pesar de la diversidad, se ha reconocido un amplio abanico de aspectos de la organización a los que contribuye la existencia de estos códigos, como los resaltados por Hoven y Brannick (1999):

- Ayudan a orientar a los nuevos empleados y fomentan un enfoque hacia un comportamiento moral. 
- Ayudan a resolver los problemas específicos de una manera concreta, propia de la organización.

- Ayudan a que los empleados piensen en la misión y en las obligaciones que tiene frente a otros trabajadores, frente a los clientes y frente a la sociedad en su conjunto.

- Su desarrollo puede servir como base para nuevos debates y aclaraciones dentro de la empresa.

- Dan seguridad a los grupos de interés al proporcionarles conocimiento sobre la existencia de unos valores de la empresa.

En definitiva, los códigos expresan la cultura de una empresa al mismo tiempo que la desarrollan. A este respecto cabe destacar que hay estudios, como el de Hoven y Brannick (1999), que han puesto de manifiesto que la cultura empresarial influye en el comportamiento de las personas más aún que su propia predisposición inicial, o como el estudio de Guiso, Sapienza y Zingales (2013), más reciente, que destaca la importancia de la cultura empresarial interna en los resultados de la gestión más que la promoción de la cultura ética en el exterior.

Aunque, los códigos éticos no están exentos de limitaciones, por ejemplo, no pueden prever todas las situaciones que pueden presentarse y en algunos casos son, fundamentalmente, un documento utilizado para trasladar una buena imagen pública de la organización. Por ello su eficacia depende, en gran medida, de su desarrollo y actualización, así como de su estricta aplicación, y de la inclusión de medidas que la empresa adopta cuando los empleados no actúan conforme al código (Ibrahim, 2012; PainterMorland, 2010; Lozano, 2007).

La UE coincide con este planteamiento y reconoce que el principal desafío que plantean los códigos de conducta reside en asegurar su aplicación, verificación y control. En este sentido, el Libro Verde de la Comisión (COM, 2001) ya reconocía las auditorías sociales como un instrumento clave para mejorar la contribución de las empresas al progreso social. En concreto, como recoge Fernández (2011), la Comisión considera necesario que los códigos de conducta tengan como normas mínimas comunes de referencia los convenios fundamentales de la OIT y las directrices de la OCDE para las empresas multinacionales; que incluyan mecanismos adecuados de evaluación y control de su aplicación, así como un sistema de evaluación de la conformidad; que impliquen en su elaboración, aplicación y control a los interlocutores sociales y otras partes interesadas, inclusive de los países en desarrollo; y anima a las empresas europeas a divulgar las experiencias y prácticas correctas en este ámbito. A lo anterior se suma el llamamiento de la Comisión para que el foro multilateral europeo sobre la RSE examinara la eficacia y credibilidad de los actuales códigos de conducta. 
Respecto al contenido de los códigos, Winkler (2011) y Holder-Webb y Cohen (2012), entre otros, concluyen que la elaboración de los códigos éticos está muy condicionada por las presiones de los grupos de interés y por las fuerzas legales, lo que da lugar al desarrollo de códigos con un contenido muy uniforme. En definitiva, en el desarrollo de los códigos lo que más influye es el deseo de legitimación ante los grupos de interés, que se lograría a través de la homogeneización de contenidos similares a los de otras organizaciones, en lugar de tratar de reflejar la singularidad moral propia, lo que supondría el desarrollo de un código específico de la empresa.

Sin embargo, en uno de los estudios más recientes sobre el tema, Bodolica y Spraggon (2015) concluyen que, aunque muchas empresas evitan dar difusión a sus códigos éticos, existe una tendencia emergente de mayor compromiso, por lo que los códigos responden cada vez más a la aspiración de construir organizaciones sostenibles basadas en valores. Como evidencia de esta afirmación demuestran que muchas empresas en Canadá están incorporando algo de originalidad en la escritura de sus códigos, rompiendo con la homogeneidad de contenidos identificada por las investigaciones precedentes. El último informe de KPMG (2014) corrobora esta idea de que los códigos éticos están desarrollándose, cada vez más, desde motivaciones intrínsecas a la organización y no tanto para cumplir con requerimientos legales. Además, el estudio destaca que su contenido ya no se limita tanto a lo que los empleados deben o no hacer, sino que se incorporan aspectos tales como la definición de objetivos a largo plazo de la organización, la visión de la empresa sobre el éxito empresarial y la definición de responsabilidades sociales.

En este mismo sentido, Ruiz-Lozano y Araque-Padilla (2013) pusieron de manifiesto la existencia de diferencias inter e intrasectoriales; así como la ausencia de valores relevantes en perspectiva con el contenido axiológico que se menciona en otros estudios.

La integración de la ética en las empresas responde a dos grandes enfoques o motivaciones, por una parte, el enfoque del cumplimiento y, por otra, el de integridad. El primer enfoque responde al objetivo de establecer un sistema de incentivos o castigos para conseguir un comportamiento determinado de las personas que integran una organización o bien para cumplir con unos requerimientos impuestos desde el exterior, bien sea el mercado o un agente regulador; el segundo enfoque responde a la motivación de conseguir el compromiso voluntario con unos valores compartidos (Lozano, 2007).

La literatura recoge evidencias de ambos enfoques que, en parte se complementan, pero que también pueden verse como un continuo vaivén de la imposición a la autorregulación (Lozano, 2007). Esta tendencia ha generado un reconocimiento creciente del código ético en el ordenamiento jurídico de 
muchos países, como un instrumento que debe promoverse en la organización empresarial para mejorar la protección de los diferentes grupos de interés, como es el caso de España, mencionado anteriormente, con la modificación de la Ley de Sociedades o del Código Penal.

A estos dos grandes enfoques se unen las motivaciones identificadas en la literatura como: el deseo de impulsar la imagen y reputación de la organización en los mercados, la obtención de legitimidad ante sus grupos de interés, la formalización de compromisos y cumplimientos legales, el deseo de reducir conflictos y costes legales mediante la promoción en la empresa de una cultura basada en la ética (Bondy et al., 2008; McDonald, 2009); e incluso en los últimos años, los códigos son vistos como una manera de responder a las recientes quiebras empresariales y evitar las consecuencias negativas de las actividades ilegales y las malas prácticas empresariales (Bodolica \& Spraggon, 2015; Singh, 2011). A todo lo anterior hay que añadir que en algunos sectores hay cierta regulación que ha promovido su desarrollo (KPMG, 2014).

Numerosos trabajos han corroborado la relación entre la existencia de un código ético en la organización y unas mejores prácticas empresariales, en línea con sus políticas de responsabilidad social, ya que se identifica una menor incidencia de las malas conductas y las organizaciones se muestran más eficaces en su detección y respuesta (Adams et al., 2001; Schwartz, 2001; KPMG, 2014).

Sin embargo, las Pymes han sido mucho más lentas en darse cuenta de estos beneficios (Del Baldo, 2006). Para Mandl (2009), Szabo (2008), Mandl y Dorr (2007) hay pruebas convincentes de que también puede ser una herramienta estratégica para mejorar la competitividad de las Pymes. En sus estudios confirman los efectos positivos en las Pymes de las prácticas de RSE, como la mejora de la productividad, la mayor satisfacción de los empleados, la mejora de la lealtad de los clientes, la disminución de los costes de operación y la mejora de la imagen de la empresa.

Pero, algunas de estas ventajas de la RSE no son evidentes para las Pymes en el corto plazo, por ejemplo, estas empresas argumentan que la reputación o gestión de la marca no es importante para ellas. Estas opiniones pueden ser consecuencia de una menor visión a largo plazo del negocio y un mayor enfoque centrado en la supervivencia a corto plazo. Para Mahmood (2008), la razón es obvia: las Pymes son más vulnerables a las crisis y se centran en la supervivencia en el corto plazo. Precisamente por todas estas dificultades, Mahmood (2008) concluye que es necesario el desarrollo de un marco de incentivos para que las Pymes se sumen al carro de la RSE.

Así, Mahmood (2008) destaca la importancia de incorporar los códigos éticos como un instrumento básico de la gestión de la RS en las Pymes y su necesaria adaptación, resaltando que el código debe ser 
un documento vivo, dinámico y flexible, es decir, derivado de una autorregulación voluntaria, adaptado a las necesidades particulares de cada organización, flexible para dar respuesta a los distintos sectores y tamaños, así como sencillo y fácil de entender. Por otro lado, señala la posibilidad de modificar la regulación existente para evitar la carga regulatoria y hacerla compatible con la existencia de estos códigos.

\subsection{Cuestiones de investigación}

La importancia creciente en la investigación a nivel internacional sobre los códigos éticos y su efectividad para conducir las relaciones con los grupos de interés favoreciendo las buenas prácticas nos ha llevado a plantearnos la primera cuestión:

- Q1. ¿Son los códigos éticos un instrumento de gestión en el tejido empresarial andaluz? En caso afirmativo, ¿'son códigos propios o responden a un modelo estandarizado?

La literatura pone de manifiesto una gran diversidad de motivaciones para el uso de los códigos éticos (Bondy et al., 2008; McDonald, 2009), de ahí que se formule la siguiente cuestión:

- Q2. ¿Cuáles son las motivaciones que han influenciado en la decisión a favor o en contra de tener un código ético?

Según Singh (2011) los códigos éticos son percibidos y organizados en la Pymes de forma distinta a las grandes empresas, así mismo, la literatura muestra que las motivaciones que llevan a las grandes empresas a implementar o desarrollar un código ético no son percibidas de la misma forma por las Pymes (Mandl \& Dorr, 2007), lo que nos lleva a plantearnos la tercera y la cuarta cuestión:

- Q3. ¿Influye el tamaño de las empresas en la tenencia de códigos éticos?

- Q4. ¿Existen diferencias significativas en las motivaciones según el tamaño de las empresas? 


\section{Metodología}

Para dar respuesta a las cuestiones planteadas en esta investigación, se ha elaborado una encuesta que permita obtener la información relacionada con la utilización de los códigos éticos por parte de las empresas con sede en Andalucía.

Para su elaboración se han utilizado los elementos principales derivados de la revisión de la literatura, y se han adaptado al objeto de la investigación; así se han definido: los tipos de códigos, las motivaciones y las posibles vías de desarrollo siguiendo entre otros a: Bondy et al. (2008), Lozano (2007), McDonald (2009) y O’Dwyer y Madden (2006).

El cuestionario consta de una pregunta para identificar la utilización del código cuya respuesta da lugar a una variable dummy, así como de un bloque de preguntas para obtener información acerca de las razones que explican su comportamiento y desarrollo, cuya valoración por parte de los encuestados se ha hecho mediante la definición de una escala de Likert de 1 a 5 (en donde 1 se identificaba con nada o totalmente en desacuerdo y 5 se identificaba con mucho o totalmente de acuerdo). Las preguntas se presentan en el análisis de los resultados.

El ámbito de estudio ha sido influenciado por la preocupación por conocer el estado de la cuestión en las empresas con las que nuestra institución se relaciona, de ahí que la población esté formada por las 2.100 empresas con sede en Andalucía que forman parte de la base de datos de la Universidad. El cuestionario se envió en los meses de marzo y abril de 2015, recibiéndose un total de 140 respuestas, de las que finalmente resultaron 116 encuestas válidas.

Las empresas de la muestra se han clasificado según tamaño atendiendo los criterios de clasificación de la recomendación de la Comisión Europea (COM, 2003)

La muestra finalmente está compuesta de 116 empresas clasificadas entre microempresas, pequeñas, medianas y grandes, según se observa en la Tabla 1.

\begin{tabular}{|l|r|r|}
\cline { 2 - 3 } \multicolumn{1}{c|}{} & $\mathbf{N}^{\mathbf{0}}$ Empresas & \% por tamaño sobre total empresas \\
\hline Microempresas & 34 & $29.3 \%$ \\
\hline Pequeñas & 25 & $21.6 \%$ \\
\hline Medianas & 22 & $19.0 \%$ \\
\hline Grandes & 35 & $30.2 \%$ \\
\hline Total & 116 & $100.0 \%$ \\
\hline
\end{tabular}

Tabla 1. Composición de la muestra clasificada por tamaño 
La metodología aplicada ha respondido a dos tipos de análisis. En primer lugar, un análisis descriptivo de los resultados del cuestionario para dar respuesta a las cuestiones 1 y 2 y, en segundo lugar, un test de diferencia de medias y un análisis de la varianza para contrastar la influencia del tamaño (cuestiones 3 y 4). Estas metodologías han sido aplicadas en estudios de naturaleza similar a éste como el de Bodolica y Spraggon (2015) y García et al. (2008).

Para responder a las cuestiones 3 y 4, en primer lugar se realizó un test de diferencia de medias para contrastar la existencia de diferencias significativas entre las Pymes y las grandes empresas a la hora de incorporar un código ético. En concreto, los resultados obtenidos para la cuestión 3 nos permitieron corroborar la existencia de diferencias por tamaño, por lo que se ha seguido profundizando, mediante comparaciones post hoc o a posteriori, lo que nos permitió identificar entre qué tamaños (microempresas, pequeñas, medianas o grandes) se producen estas diferencias significativas (Kvanli, Pavur \& Keeling, 2003).

\section{Resultados}

En relación a la tenencia de códigos éticos por parte de las entidades analizadas, de las 116 empresas, 76 empresas han manifestado que poseen código ético (un $65.5 \%$ de la muestra) frente a 40 empresas que declaran no poseer código ético en este momento (34.5\%) (Tabla 2).

Entre las empresas que poseen código ético destacar que son más del 80 \% de las empresas medianas y grandes analizadas, y que en el caso de las microempresas son más del 60\% las que lo poseen.

Entre las empresas que no poseen código ético es de señalar que 32 de las 40 empresas son microempresas o pequeñas empresas.

\begin{tabular}{|l|r|r|r|r|r|}
\hline & \multicolumn{2}{|c|}{ Código Si } & \multicolumn{2}{c|}{ Código No } & \multicolumn{1}{c|}{ Total } \\
\hline Microempresas & 21 & $61.8 \%$ & 13 & $38.2 \%$ & 34 \\
\hline Pequeñas & 6 & $24.0 \%$ & 19 & $76.0 \%$ & 25 \\
\hline Medianas & 18 & $81.8 \%$ & 4 & $18.2 \%$ & 22 \\
\hline Grandes & 31 & $88.6 \%$ & 4 & $11.4 \%$ & 35 \\
\hline Total & 76 & $65.5 \%$ & 40 & $34.5 \%$ & 116 \\
\hline
\end{tabular}

Tabla 2. Empresas que tienen código ético

Para analizar si la falta de código ético es o no una cuestión puntual se ha solicitado a los encuestados una valoración de la probabilidad de que tengan intención de incorporarlo a su gestión en un periodo 
de medio plazo y, por tanto, una aproximación a la posibilidad de que en este momento estén trabajando en ello, poniéndose de manifiesto (ver Tabla 3) que la probabilidad es baja, es decir, son pocas las empresas que se lo están planteando. Pero llama la atención que el valor más alto se presenta en las empresas pequeñas, que son precisamente las que tenían una mayor presencia en la falta de incorporación de códigos, lo que nos lleva a pensar que existe una cierta tendencia a la incorporación de los códigos éticos en todo tipo de empresas.

\begin{tabular}{|r|c|r|r|}
\hline Microempresas & Pequeñas & Medianas & Grandes \\
\hline $2.4(1.67)$ & $3.1(1.55)$ & $2.3(0.50)$ & $2.8(1.26)$ \\
\hline
\end{tabular}

Tabla 3. Intención de tener un código ético a medio plazo, valoración media* *Valores medios por tipos de empresas clasificadas por tamaño y entre paréntesis se expresa la desviación típica

En relación a los motivos que inducen a que las empresas de la muestra no posean un código ético observamos (Tabla 4) que no hay una valoración clara que destaque entre los motivos propuestos, ya que los valores que se presentan en todo tipo de empresas son más bien bajos. Predomina algo la consideración de que no se ha pensado en ello, salvo en el caso de las grandes empresas que como se ha mencionado en la literatura previa se ven presionadas por el entorno e incluso por los cambios en la regulación legislativa. Así mismo, es de señalar que los valores medios más bajos se presentan en la consideración de que no sirvan para nada, de lo que se deduce que existe una cierta creencia de que puede ser un instrumento que puede aportar a la mejora de la gestión empresarial aunque algunas empresas no tengan muy claro cómo.

\begin{tabular}{|l|r|r|r|r|}
\hline \multicolumn{1}{|c|}{ Motivos } & Microempresas & \multicolumn{1}{c|}{ Pequeñas } & \multicolumn{1}{c|}{ Medianas } & \multicolumn{1}{c|}{ Grandes } \\
\hline No lo ven necesario & $2.85(1.52)$ & $2.25(1.36)$ & $3(1)$ & $3(2.31)$ \\
\hline No tienen tiempo & $2.27(1.27)$ & $2.7(1.49)$ & $2.67(1.53)$ & $2(1.15)$ \\
\hline Creen que no sirve para nada & $1.6(1.26)$ & $1.33(0.65)$ & $1.67(1.15)$ & $1.5(1)$ \\
\hline No han pensado en ello & $3.23(1.59)$ & $2.86(1.41)$ & $3.25(1.71)$ & $1.75(0.96)$ \\
\hline No creen que sea aplicable a una Pyme & $2(1.41)$ & $1.5(0.90)$ & $3.25(1.71)$ & $1.75(0.96)$ \\
\hline
\end{tabular}

Tabla 4. Valoración media de los motivos por los que no tienen código ético*

*Valores medios por tipos de empresas clasificadas por tamaño y entre paréntesis se expresa la desviación típica

En cuanto a las empresas que han manifestado tener códigos éticos, interesa conocer el tipo de código que se está utilizando, si se trata de un modelo propio o de un modelo estándar, como consecuencia de la importancia que los estudios previos han dado a la necesidad de que sea un instrumento adaptado a las particularidades de las empresas para dotarlo de mayor efectividad. Los resultados obtenidos 
(Tabla 5) ponen de manifiesto que la mayor parte de las empresas medianas y grandes han hecho el esfuerzo por desarrollar su propio código, mientras que en el caso de las micro y pequeñas empresas están utilizando, o bien un modelo propio o bien un modelo del sector, en ningún caso un modelo estándar.

\begin{tabular}{|l|r|r|r|r|}
\cline { 2 - 5 } \multicolumn{1}{c|}{} & \multicolumn{1}{c|}{ Microempresas } & Pequeñas & \multicolumn{1}{c|}{ Medianas } & \multicolumn{1}{c|}{ Grandes } \\
\hline Modelo sectorial o profesional & 46.2 & 33.3 & 0 & 10.7 \\
\hline Modelo estándar & 0 & 0 & 5.9 & 3.6 \\
\hline Modelo propio & 53.8 & 66.7 & 94.1 & 85.7 \\
\hline
\end{tabular}

Tabla 5. Porcentaje de presencia de los diferentes tipos de código ético

A las empresas que han desarrollado su propio código se les ha requerido una valoración de cómo ha sido el proceso de desarrollo, poniéndose de manifiesto en los resultados (Tabla 6) que, en general, se ha valorado el liderazgo asumido por la dirección, destacando el caso del $100 \%$ de las pequeñas empresas.

Así mismo resalta la valoración de la influencia que ha tenido en su desarrollo la política empresarial, con valores medios en torno a 4. Mientras que, por el contrario, destaca cómo los procesos han sido poco participativos, tanto por parte de los empleados como de otros grupos de interés, salvo en el caso de las pequeñas en donde un valor medio cercano a 4 y con baja desviación típica nos lleva a pensar que, de alguna forma, se ha contado con la participación de los empleados.

\begin{tabular}{|c|c|c|c|c|}
\hline Código propio & Microempresas & Pequeñas & Medianas & Grandes \\
\hline Está desarrollado a partir del análisis de la política empresarial & $4.4(1.06)$ & $4.3(0.5)$ & $3.9(1.33)$ & $3.8(1.18)$ \\
\hline Se ha desarrollado con la participación de los empleados & $3.2(1.56)$ & $4(0.81)$ & $2.73(1.28)$ & $2.8(1.23)$ \\
\hline Se ha desarrollado con la participación de otros grupos de interés & $2(1.15)$ & $3(2)$ & $2.8(1.32)$ & $2.96(1.19)$ \\
\hline Está liderado por la dirección & $4.4(1.41)$ & $5(0)$ & $4.67(0.72)$ & $4.7(0.67)$ \\
\hline
\end{tabular}

Tabla 6. Cómo se ha desarrollado el código propio*

*Valores medios por tipos de empresas clasificadas por tamaño y entre paréntesis se expresa la desviación típica

En relación a los códigos sectoriales o profesionales son sólo 11 entidades las que lo han tenido en cuenta para su incorporación en su organización. Para tratar de detectar los motivos que le han llevado a su consideración o la falta de ello se pidió a los encuestados que valoraran el código sectorial o profesional que les afectaba (Tabla 7). Destaca la alta valoración por parte de las pequeñas empresas de los códigos sectoriales, aunque creen que no están siendo considerados por las empresas del sector, poniendo en duda su utilidad (valoración que ha sido reforzada por la opinión del resto de los tipos de empresas). 


\begin{tabular}{|l|r|r|r|r|}
\hline \multicolumn{1}{|c|}{ Código sectorial o profesional } & Microempresas & Pequeñas & Medianas & Grandes \\
\hline Considero que el código responde a las necesidades de mi empresa & $3.5(1.04)$ & $4.5(0.71)$ & $3.7(0.58)$ & $3.7(1.1)$ \\
\hline Considero que responde a los aspectos críticos del sector & $3.7(1.03)$ & $4.7(0.58)$ & $3.7(0.58)$ & $3.6(0.92)$ \\
\hline Considero que el código es respetado por las empresas del sector & $2.3(1.21)$ & $2.3(1.53)$ & $3(1)$ & $3.4(1.07)$ \\
\hline
\end{tabular}

Tabla 7. Valoración media del código sectorial o profesional*

*Valores medios por tipos de empresas clasificadas por tamaño y entre paréntesis se expresa la desviación típica

La literatura pone de manifiesto la diversidad de motivaciones que pueden estar afectando a la incorporación del código ético como instrumento de gestión de la RS. Diversidad que es corroborada por los resultados obtenidos (Tabla 8). La mayor parte de las motivaciones propuestas han sido muy valoradas con independencia del tamaño de la empresa. Sin embargo, destacan motivaciones internas como el que se considere un documento inspirador de la cultura empresarial y especialmente enfocado en orientar el comportamiento de los empleados (motivaciones 11 y 12); y motivaciones externas como la transparencia y comunicación hacia los grupos de interés, lo que incide en la generación de confianza en los diferentes grupos de interés (motivaciones 3 y 4). Por otro lado, es de destacar, que los valores más bajos ponen de manifiesto una falta de reconocimiento de la influencia del entorno actual salvo en el caso de las grandes empresas.

Si tenemos en cuenta el tamaño, es de destacar como las empresas medianas no creen que incida en ello el que lo puedan tener las empresas de la competencia y que las empresas pequeñas no perciben que les pueda ayudar a gestionar los riesgos, posiblemente como consecuencia de que sus riesgos son más de carácter económico-financiero que reputacionales.

\begin{tabular}{|c|c|c|c|c|}
\hline Motivos & Microempresas & Pequeñas & Medianas & Grandes \\
\hline 1. Asegurar el cumplimiento legal & $3.3(1.28)$ & $3(0)$ & $3.6(1.5)$ & $3.9(1.18)$ \\
\hline 2. Mejorar nuestra reputación & $3.8(1.40)$ & $4(1.15)$ & $3.7(0.98)$ & 3.901 .02 \\
\hline 3. Diferenciar nuestros productos/servicios & $4.3(1.04)$ & $3.4(0.89)$ & $4.2(1.01)$ & $4.2(1)$ \\
\hline 4. Generar confianza en los grupos de interés & $4.3(1.0)$ & $4(1.1)$ & $4.5(0.89)$ & $4.2(0.99)$ \\
\hline 5. Generar mejor el riesgo & $4(1.10)$ & $2.5(1)$ & $3.9(1.55)$ & $4.1(1.16)$ \\
\hline 6. Regular el comportamiento similar en todas las filiales de la empresa & $4.3(1.04)$ & $3.5(1.91)$ & $4.3(1.27)$ & $4.2(1.15)$ \\
\hline 7. Comunicar los principios y compromisos a los grupos de interés & $4.3(1)$ & $3.5(1.91)$ & $4.6(0.81)$ & $4.4(0.94)$ \\
\hline 8. Otras empresas del sector tienen uno & $3(1.63)$ & $3(2.83)$ & $1(0)$ & $3(2)$ \\
\hline 9. Me obliga el entorno actual & $2.3(1.03)$ & $3(0)$ & $2.5(0.93)$ & $3.5(0.89)$ \\
\hline $\begin{array}{l}\text { 10. Disminuir el impacto en el medio ambiente y contribuir } \\
\text { socialmente }\end{array}$ & $3.8(1.04)$ & $4(1.15)$ & $4.3(1.0)$ & $3.6(0.95)$ \\
\hline $\begin{array}{l}\text { 11. Generar una cultura interna de respeto, desarrollo y de } \\
\text { cooperación }\end{array}$ & $4.2(1.01)$ & $4.5(1)$ & $4.5(0.87)$ & $4.5(0.90)$ \\
\hline 12. Guiar a los empleados en la toma de decisiones éticas & $4.2(1.03)$ & $4(1.1)$ & $4.4(0.93)$ & $4.4(0.93)$ \\
\hline
\end{tabular}

Tabla 8. Valoración de los motivos por los que dispone de un código ético* *Valores medios por tipos de empresas clasificadas por tamaño y entre paréntesis se expresa la desviación típica

Para asegurar el que las respuestas de los encuestados se derivaran de su conocimiento en relación con el tema analizado, en algunas de las preguntas que forman parte de la encuesta se ha incorporado la 
opción NS/NC (no sabe/no contesta). Esta opción, de forma general, ha sido de las menos seleccionadas por los encuestados, lo que puede indicar en gran medida, que las personas que han respondido la encuesta son conocedoras de los planteamientos relacionados con el desarrollo e implantación del código ético en su empresa y, proporciona a los resultados obtenidos mayor fiabilidad.

Las cuestiones en las que el encuestado opta por elegir NS/NC están relacionadas principalmente con:

- El motivo por el que no se dispone de código ético.

- Otros aspectos relacionados con los códigos éticos de las empresas que si lo tienen implantado.

En el primer caso, los encuestados demuestran no tener claro por qué su empresa no dispone de código ético (Tabla 9), no se decantan por afirmaciones tales como: que no lo ven necesario, o que no disponen de tiempo para su desarrollo o simplemente que no han pensado en ello. Las frecuencias más altas donde se opta por NS/NC están en torno al 14.5\%, donde el encuestado manifiesta no saber si el código ético aporta valor al negocio del código o si es susceptible de aplicación en la Pyme.

\begin{tabular}{|r|r|r|r|r|}
\hline $\begin{array}{c}\text { No lo vemos necesario } \\
\text { en nuestra empresa }\end{array}$ & $\begin{array}{c}\text { No tenemos } \\
\text { tiempo }\end{array}$ & $\begin{array}{c}\text { Creemos que un código ético } \\
\text { no aporta valor al negocio }\end{array}$ & $\begin{array}{c}\text { No hemos } \\
\text { pensado en ello }\end{array}$ & $\begin{array}{c}\text { No creemos que sea } \\
\text { aplicable a una PYME }\end{array}$ \\
\hline 13.51 & 12.50 & 14.71 & 10.26 & 14.29 \\
\hline
\end{tabular}

Tabla 9. Razones por las que no se dispone de código ético: \% de NS/NC sobre el total de las respuestas obtenidas

Cuando la empresa dispone de código propio, destaca la falta de conocimiento sobre cómo la posible participación en su desarrollo por parte de otros grupos de interés que no sean los empleados, y es llamativo como los encuestados no tienen claro si las empresas del sector disponen de un código propio, o si existiendo el mismo, el resto de las empresas del sector lo respetan, ni siquiera si el código responde a las necesidades de su empresa. Estos porcentajes superan el $20 \%$ de las respuestas obtenidas, como puede apreciarse en la Tabla 10.

\begin{tabular}{|r|c|c|c|c|}
\hline $\begin{array}{c}\text { Se ha desarrollado } \\
\text { con la participación } \\
\text { de otros grupos de } \\
\text { interés }\end{array}$ & $\begin{array}{c}\text { Considero que el } \\
\text { código responde a } \\
\text { las necesidades de } \\
\text { mi empresa }\end{array}$ & $\begin{array}{c}\text { Considero que } \\
\text { responde a los } \\
\text { aspectos críticos del } \\
\text { sector }\end{array}$ & $\begin{array}{c}\text { Considero que el } \\
\text { código es respetado } \\
\text { por las empresas del } \\
\text { sector }\end{array}$ & $\begin{array}{c}\text { La empresa dispone } \\
\text { de un código ético } \\
\text { porque las empresas } \\
\text { del sector tienen uno }\end{array}$ \\
\hline 10.71 & 21.43 & 20.69 & 24.14 & \\
\hline
\end{tabular}

Tabla 10. En caso de código propio: \% de NS/NC sobre el total de las respuestas obtenidas

De cuyo análisis se deriva la falta de conocimiento sobre el comportamiento de la competencia y de otras empresas del sector. 


\subsection{Influencia del tamaño empresarial}

En primer lugar, se analizó esta cuestión entre los dos principales grupos de empresas, las Pymes y las grandes empresas. Los resultados de la Tabla 11 corroboran la existencia de diferencias significativas entre el número de Pymes que disponen de un código ético y el número de grandes empresas, confirmando la hipótesis de que el tamaño empresarial influye en la decisión de incorporar un código ético o no en la gestión.

\begin{tabular}{|c|c|c|c|c|c|c|c|c|}
\hline \multicolumn{2}{|c|}{$\begin{array}{l}\text { Prueba de Levene de } \\
\text { calidad de varianzas }\end{array}$} & \multicolumn{7}{|c|}{ Prueba t para la igualdad de medias } \\
\hline \multirow[t]{2}{*}{$\mathbf{F}$} & \multirow[t]{2}{*}{ Sig. } & \multirow[t]{2}{*}{$\mathbf{t}$} & \multirow[t]{2}{*}{ gl } & \multirow[t]{2}{*}{$\begin{array}{c}\text { Sig. } \\
\text { (bilateral) }\end{array}$} & \multirow[t]{2}{*}{$\begin{array}{l}\text { Diferencia } \\
\text { de medias }\end{array}$} & \multirow[t]{2}{*}{$\begin{array}{l}\text { Diferencia de } \\
\text { error estándar }\end{array}$} & \multicolumn{2}{|c|}{$\begin{array}{l}95 \% \text { de intervalo de } \\
\text { confianza de la } \\
\text { diferencia }\end{array}$} \\
\hline & & & & & & & Inferior & Superior \\
\hline 105.826 & .000 & -4.484 & 114 & .000 & -.4189 & .0934 & -.6040 & -.2338 \\
\hline
\end{tabular}

Tabla 11. Test de diferencia de medias: Pymes versus grandes

Los resultados anteriores únicamente permiten contrastar la hipótesis general de que los promedios comparados no son iguales entre las Pymes y las grandes empresas. Con el objetivo de seguir profundizando en estas diferencias por tamaño, se realizaron los contrastes denominados comparaciones múltiples post hoc o comparaciones a posteriori entre microempresas, pequeñas, medianas y grandes empresas. Previo al análisis post hoc se ha realizado la prueba de homogeneidad de la varianza, obteniendo como resultados que la varianza no es homogénea por lo que se utilizó el método de Games Howell, que es el de mayor aceptación en caso de varianza no homogénea (Kvanli et al., 2003). Los resultados se recogen en la Tabla 12. 


\begin{tabular}{|c|c|c|c|c|c|c|}
\hline & & \multirow{2}{*}{$\begin{array}{l}\text { Diferencia de } \\
\text { medias (I-J) }\end{array}$} & \multirow{2}{*}{$\begin{array}{c}\text { Error } \\
\text { estándar }\end{array}$} & \multirow{2}{*}{ Sig. } & \multicolumn{2}{|c|}{$\begin{array}{l}95 \% \text { de intervalo de } \\
\text { confianza }\end{array}$} \\
\hline & & & & & $\begin{array}{l}\text { Límite } \\
\text { inferior }\end{array}$ & $\begin{array}{c}\text { Límite } \\
\text { superior }\end{array}$ \\
\hline \multirow{3}{*}{ Microempresas } & Pequeñas & .1424 & .1215 & .647 & -.179 & .464 \\
\hline & Medianas & $-.4358^{*}$ & .1193 & .003 & -.753 & -.119 \\
\hline & Grandes & $-.5034^{*}$ & .1007 & .000 & -.770 & -.237 \\
\hline \multirow{3}{*}{ Pequeñas } & Microempresas & -.1424 & .1215 & .647 & -.464 & .179 \\
\hline & Medianas & $-.5782^{*}$ & .1212 & .000 & -.901 & -.255 \\
\hline & Grandes & $-.6457^{*}$ & .1028 & .000 & -.921 & -.371 \\
\hline \multirow{3}{*}{ Medianas } & Microempresas & $.4358^{*}$ & .1193 & .003 & .119 & .753 \\
\hline & Pequeñas & $.5782^{*}$ & .1212 & .000 & .255 & .901 \\
\hline & Grandes & -.0675 & .1003 & .907 & -.337 & .202 \\
\hline \multirow{3}{*}{ Grandes } & Microempresas & $.5034^{*}$ & .1007 & .000 & .237 & .770 \\
\hline & Pequeñas & $.6457^{*}$ & .1028 & .000 & .371 & .921 \\
\hline & Medianas & .0675 & .1003 & .907 & -.202 & .337 \\
\hline
\end{tabular}

Tabla 12. Comparaciones post hoc según tamaño empresarial

Los resultados de la Tabla 12 muestran que existen diferencias estadísticamente significativas entre:

- Las microempresas y las empresas medianas y grandes.

- Entre las empresas pequeñas y las medianas y grandes.

- No existen diferencias significativas entre las microempresas y pymes, ni entre medianas y grandes entre sí.

Una vez corroborada la influencia del tamaño en la decisión de incorporar o no un código ético, se ha seguido profundizando en la repercusión de esta variable en otros aspectos tratados en esta investigación, como son el tipo de código y los motivos que han influido en la decisión a favor o en contra de tenerlo.

En esta segunda parte del análisis se han encontrado diferencias significativas por tamaño en cuanto al tipo de código, según muestran los resultados de la Tabla 13, como se ha expuesto en el análisis descriptivo las empresas medianas y grandes han optado mayormente por la incorporación de códigos propios mientras que las micro y pequeñas empresas hacen un mayor uso de modelos comunes.

También se han encontrado diferencias significativas por tamaño en el papel que el entorno ha tenido a la hora de incorporar un código ético como una de las motivaciones (Tabla 13). 


\begin{tabular}{|l|l|r|r|r|r|r|}
\cline { 3 - 6 } \multicolumn{2}{c|}{} & Suma de cuadrados & \multicolumn{1}{c|}{ g1 } & Media cuadrática & \multicolumn{1}{c|}{ F } & Sig. \\
\hline \multirow{2}{*}{$\begin{array}{l}\text { Pregunta 5. Tipo de } \\
\text { código }\end{array}$} & Entre grupos & 6.552 & 3 & 2.184 & 4.304 & .008 \\
\cline { 2 - 7 } & Dentro de grupos & 30.448 & 60 & & .507 & \\
\cline { 2 - 7 } & Total & 37.000 & 63 & 3.275 & 3.896 & .018 \\
\hline $\begin{array}{l}\text { Pregunta 8.9. } \\
\text { Influencia del } \\
\text { entorno }\end{array}$ & Entre grupos & 9.825 & 3 & & .841 & \\
\cline { 2 - 7 } & Dentro de grupos & 26.061 & 31 & & & \\
\cline { 2 - 7 } & Total & 35.886 & 34 & & & \\
\hline
\end{tabular}

Tabla 13. Resultados ANOVA. Tipos de códigos e influencia del entorno

Para profundizar en el análisis y conocer entre qué grupos se dan esas diferencias se ha realizado el análisis post hoc, poniéndose de manifiesto que en relación a los tipos de códigos las diferencias se dan entre las microempresas y las empresas medianas y grandes (Tabla 14). Mientras que en el análisis de la influencia del entorno se muestran las diferencias entre las empresas medianas y las grandes, aunque también podría afirmarse que existen diferencias entre las microempresas y las grandes (Tabla 14). Por lo que podríamos concretar que las grandes reconocen que se han visto más influidas por el entorno a la hora de incorporar un código ético en su gestión.

\begin{tabular}{|c|c|c|c|c|c|c|c|}
\hline \multirow{2}{*}{\multicolumn{3}{|c|}{ Variable dependiente }} & \multirow{3}{*}{$\begin{array}{r}\text { Diferencias de } \\
\text { medias (I-J) } \\
-.2564\end{array}$} & \multirow{3}{*}{$\begin{array}{r}\begin{array}{c}\text { Error } \\
\text { estándar }\end{array} \\
.3516 \\
\end{array}$} & \multirow{3}{*}{$\begin{array}{l}\text { Sig. } \\
.885 \\
\end{array}$} & \multicolumn{2}{|c|}{$95 \%$ de intervalo de confianza } \\
\hline & & & & & & \multirow{2}{*}{$\begin{array}{r}\text { Límite inferior } \\
-1.185\end{array}$} & \multirow{2}{*}{\begin{tabular}{|r|} 
Límite superior \\
.673 \\
\end{tabular}} \\
\hline \multirow{12}{*}{$\begin{array}{l}\text { Pregunta } 5 . \\
\text { Tipo de } \\
\text { código }\end{array}$} & \multirow{3}{*}{ Micro } & Pequeñas & & & & & \\
\hline & & Medianas & $-.8643^{*}$ & .2625 & .009 & -1.558 & -.171 \\
\hline & & Grandes & $-.6731 *$ & .2391 & .032 & -1.305 & -.041 \\
\hline & \multirow{3}{*}{ Pequeñas } & Micro & .2564 & .3516 & .885 & -.673 & 1.185 \\
\hline & & Medianas & -.6078 & .3383 & .285 & -1.502 & .286 \\
\hline & & Grandes & -.4167 & .3205 & .566 & -1.264 & .430 \\
\hline & \multirow{3}{*}{ Medianas } & Micro & $.8643^{*}$ & .2625 & .009 & .171 & 1.558 \\
\hline & & pequeñas & .6078 & .3383 & .285 & -.286 & 1.502 \\
\hline & & Grandes & .1912 & .2190 & .819 & -.388 & .770 \\
\hline & \multirow{3}{*}{ Grandes } & \begin{tabular}{|l} 
Micro \\
\end{tabular} & $.6731 *$ & .2391 & .032 & .041 & 1.305 \\
\hline & & Pequeñas & .4167 & .3205 & .566 & -.430 & 1.264 \\
\hline & & Medianas & -.1912 & .2190 & .819 & -.770 & .388 \\
\hline \multirow{12}{*}{$\begin{array}{l}\text { Pregunta } \\
8.9 \text {. } \\
\text { Influencia } \\
\text { del entorno }\end{array}$} & \multirow{3}{*}{ Micro } & Pequeñas & -.6667 & .7486 & .810 & -2.698 & 1.365 \\
\hline & & Medianas & -.1212 & .4653 & .994 & -1.384 & 1.142 \\
\hline & & Grandes & -1.1667 & .4389 & .057 & -2.358 & .025 \\
\hline & \multirow{3}{*}{ Pequeñas } & \begin{tabular}{|l|} 
Micro \\
\end{tabular} & .6667 & .7486 & .810 & -1.365 & 2.698 \\
\hline & & Medianas & .5455 & .7048 & .866 & -1.367 & 2.458 \\
\hline & & Grandes & -.5000 & .6877 & .885 & -2.366 & 1.366 \\
\hline & \multirow{3}{*}{ Medianas } & Micro & .1212 & .4653 & .994 & -1.142 & 1.384 \\
\hline & & pequeñas & -.5455 & .7048 & .866 & -2.458 & 1.367 \\
\hline & & Grandes & $-1.0455^{*}$ & .3591 & .032 & -2.020 & -.071 \\
\hline & \multirow{3}{*}{ Grandes } & Micro & 1.1667 & .4389 & .057 & -.025 & 2.358 \\
\hline & & Pequeñas & .5000 & .6877 & .885 & -1.366 & 2.366 \\
\hline & & Medianas & $1.0455^{*}$ & .3591 & .032 & .071 & 2.020 \\
\hline
\end{tabular}

Tabla 14. Comparaciones post hoc Tipos de códigos e influencia del entorno 
Se ratifica la cuestión 3 en cuanto a la influencia del tamaño empresarial en la tenencia de códigos éticos y el modelo que incorporan. Pero en relación a la cuestión 4 sólo hemos podido encontrar diferencias significativas por el tamaño en una de las motivaciones, la del papel que está teniendo el entorno.

\section{Conclusiones y limitaciones del estudio}

En los últimos años se ha observado un creciente interés por la incorporación de los códigos éticos en la gestión de las empresas guiado por la necesidad de responder a los nuevos marcos regulatorios o presión del entorno, o bien para responder a la necesidad de orientar el comportamiento de las organizaciones hacia la integridad. Esta preocupación trasladada al ámbito de las líneas de investigación de nuestra institución y centrada en las relaciones con los grupos de interés de nuestra universidad ha orientado esta investigación.

Los resultados han detectado una cierta tendencia, en las empresas de la muestra, a la incorporación de los códigos éticos como instrumento de gestión de los valores de las organizaciones empresariales y de las políticas de responsabilidad social en los casos que corresponda.

En el desarrollo de los códigos ha predominado la incorporación de códigos propios, en línea con resultados obtenidos por Bodolica y Spraggon (2015). Se ha valorado el que los procesos han sido liderados por la dirección, pero también se ha destacado que han sido procesos poco participativos, por lo que habría que trabajar en la línea de incorporar a los grupos de interés en el futuro desarrollo de los códigos.

Entre las motivaciones que han conducido el desarrollo de los códigos recibe una valoración máxima por parte de todas las empresas la utilidad para afianzar la cultura interna, posiblemente por el reconocimiento de su mayor incidencia en la gestión como mostraron los resultados obtenidos por Guiso et al. (2013)

Los resultados relacionados con la utilización de modelos sectoriales, sobre todo por parte de las micro y pequeñas empresas, ponen de manifiesto la importancia de ampliar los esfuerzos en el desarrollo de modelos de códigos éticos por sectores empresariales porque supone un primer avance hacia la adaptación a las circunstancias particulares de la actividad empresarial y, por tanto, un paso hacia la efectividad de los códigos en el caso de que no haya recursos suficientes para desarrollar su propio código. Al mismo tiempo, señalamos la necesidad de una mayor comunicación de estos códigos porque se ha observado un cierto desconocimiento por parte de los encuestados de este tipo de códigos y del 
comportamiento del sector en este sentido. Por otro lado, el desconocimiento de la competencia nos lleva a pensar que estas empresas han centrado su atención en grupos de interés como son los empleados y los clientes.

Así mismo, señalamos que la falta de una valoración clara de los motivos que les han llevado a no tener un código ético nos induce a especular que hay una falta de preocupación o reflexión al respecto, posiblemente por desconocimiento del instrumento o por la atención prestada a otros aspectos claves de su gestión y, por tanto, a plantear la necesidad de una mayor formación en este ámbito.

Se ha puesto de manifiesto la presencia de diferencias significativas por tamaño en la existencia o no de código, así como en el tipo de código si se posee. Un análisis más profundo nos permite concluir que existen tres grandes grupos al analizar la influencia del tamaño empresarial: microempresas y pequeñas, medianas y grandes empresas, no sólo dos como se ha venido diferenciando en la literatura analizada, ya que las medianas en algunos casos se asemeja más a las grandes que a las pequeñas. En todo caso, se confirma la diferente aproximación a la RS de cada grupo, de acuerdo con Russo y Perrini, (2010)

Por otra parte, las micro y las pequeñas empresas han incorporado el código para responder al interés del propietario mientras que las grandes se dejan influenciar por la regulación de las multinacionales y de las cotizadas, en contra de las medianas que no se encuentran motivadas por ninguno de estos aspectos.

Todo ello nos lleva a plantear la necesidad de incentivar y ayudar no sólo a las micro y pequeñas empresas, sino de prestar una mayor atención a las medianas por su carácter diferenciado respecto a los otros grupos.

El entorno también ha tenido un protagonismo diferente, entre las micro, las medianas y grandes empresas, a la hora de tomar la decisión de incorporar un código ético, poniéndose de manifiesto su influencia regulatoria o el enfoque del criterio de cumplimiento.

Entre las limitaciones de este estudio destaca su carácter exploratorio en el ámbito andaluz por las restricciones de la base de datos y del tamaño de la muestra, pero que nos ha permitido acercarnos a la realidad y tener una aproximación de lo que está ocurriendo en este terreno.

Futuras investigaciones nos permitirán ampliar nuestro conocimiento al considerar otras bases de datos. Por otro lado, dado que no se han encontrado diferencia de medias significativas entre las motivaciones por las que las empresas hacen o no uso de los códigos como instrumento de gestión de la 
responsabilidad social habría que explorar qué otras variables económico-financieras o de gestión de la actividad pueden estar influyendo.

\section{Agradecimientos}

Este trabajo se ha desarrollado con el apoyo del Proyecto de Investigación de Excelencia de la Junta de Andalucía, SEJ-6073, La protección de los consumidores como ariete de la reforma de la empresa, las reglas de competencia y la cultura empresarial, cuyo investigador principal es el Prof. Dr. Juan Ignacio Font Galán.

\section{References}

Adams, J., Tasachian, A., \& Stone, T. (2001). Codes of Ethics as Signals for Ethical Behavior. Journal of Business Ethics, 29, 199-211. http://dx.doi.org/10.1023/A:1026576421399

Bodolica, V., \& Spraggon, M. (2015). An Examination into the Disclosure, Structure and Contents of Ethical Codes in Publicly Listed Acquiring Firms. Journal of Business Ethics, 126, 459-472. http://dx.doi.org/10.1007/s10551-013-1966-x

Bondy, K., Matten, D., \& Moon, J. (2008). Codes of Conduct as a Tool for Sustainable Governance in MNCs. En A. Crane, D. Matten \& L. Spence (Eds.), Corporate Social Responsibility. Readings and cases in a global context. New York: Routledge.

COM (2001). Fomentar un Marco Europeo para la Responsabilidad Social de las Empresas, Libro Verde. Comisión Europea. Disponible online en: http://goo.gl/W6ZL4a

COM (2003). Recomendación 2003/361/CE de la Comisión, de 6 de mayo de 2003, sobre la definición de pequeñas y medianas empresas (DOCE, L124, de 20 de mayo de 2003). Disponible online en: http://goo.gl/U8kJzv

Del Baldo, M. (2006). SMEs and Corporate Social Responsibility. Some evidences from an empirical research. Proceedings on emerging issues in international accounting \& business conference, 1: 314-343. University of Padua.

Fernández, I. (2011). La Dimensión Económica del Desarrollo Sostenible. Alicante: ECU, Editorial Club Universitario. 
García, I.M., Rodríguez, L., \& Gallego, I. (2008). La Influencia del Consejo de Administración en la Adopción de un Código Ético. Spanish Accounting Review, 11(2), 93-120.

Guiso, L., Sapienza, P., \& Zingales, L. (2013). The Value of Corporate Culture. Disponible online en https://goo.gl/y6hQNL

Holder-Webb, L., \& Cohen, J. (2012). The Cut and Paste Society: Isomorphism in Codes of Ethics. Journal of Business Ethics, 107, 485-509. http://dx.doi.org/10.1007/s10551-011-1060-1

Hoven, J., \& Brannick, T. (1999). Code and Conduct: Predictors of Irish Managers' Ethical Reasoning. Journal of Business Ethics, 22, 311-326. http://dx.doi.org/10.1023/A:1006017724977

Ibrahim, N. (2012). Business Versus Non-Business Students Perceptions' of Business Codes of Ethics. Proceedings of ASBBS Annual Conference: Las Vegas, 635-643.

Jenkins, R. (2001). Corporate Codes of Conduct. Self-Regulation in a Global Economy. Technology, Business and Society. Programme Paper Number 2. Disponible online en: http://www.unrisd.org/unrisd/website/document.nsf/240da49ca467a53f80256b4f005ef245/e3b3e78bab9a886f80256b 5e00344278/\$FILE/ienkins.pdf

Kvanli, A.H., Pavur, R.J., \& Keeling, K.B. (2003). Introduction to Business Statistics: A Microsoft Excel Integrated Approach. South-Western College Pub.

Kaptein, M. (2004). Business Codes of Multinational Firms: What Do They Say?. Journal of Business Ethics, 50, 13-31. http://dx.doi.org/10.1023/B:BUSI.0000021051.53460.da

KPMG (2014). Business Codes of the Global 200: What the largest companies in the world say and do. (KPMG, Netherlands). Disponible online en: https://www.kpmg.com/BE/en/IssuesAndInsights/ArticlesPublications/Documents/The-Business-codes-of-theFortune-Global-200.pdf

Lozano, J.F. (2007). Códigos Éticos y Auditorías Éticas. Véritas, 17, 225-251.

McDonald, G.M. (2009). An Anthology of Codes of Ethics. European Business Review, 21, 344-372. http://dx.doi.org/10.1108/09555340910970445

Mahmood, S. (2008). Corporate Governance and Business Ethics for SMEs in Developing Countries, Challenges and Way Forward. ISBEE World Congress 15-18 July.

Mandl, I. (2009). The Interaction between Local Employment Development and Corporate Social Responsibility. Vienna: Austrian Institute for SME Research, Elise Crunel.

Mandl, I., \& Dorr, A. (2007). CSR and competitiveness. European SMEs' good practice. Consolidated European Report, Vienna. 
Melé, D. (1999). Tres Versiones de la Ética Empresarial. Barcelona: IESE. Universidad de Navarra. Disponible online en: http://www.eticaed.org/10.Melé01.pdf

O'dwyer, B., \& Madden, G. (2006). Ethical Codes of Conduct in Irish Companies: A Survey of Code Content and Enforcement Procedures. Journal of Business Ethics, 63, 217-236. http://dx.doi.org/10.1007/s10551-005-3967-x

Painter-Morland, M. (2010). Questioning Corporate Codes of Ethics. Business Ethics: A European Review, 19, 265-279. http://dx.doi.org/10.1111/j.1467-8608.2010.01591.x

Preuss, L. (2009). Ethical Sourcing Codes of Large UK- Based Corporations: Prevalence, Content, Limitations. Journal of Business Ethics, 88, 735-747. http://dx.doi.org/10.1007/s10551-008-9978-7

Rezaee, Z., Elmore, R., \& Szendi, J. (2001). Ethical Behaviour in Higher Education Institutions: The Role of the Code of Conduct. Journal of Business Ethics, 30(2), 171-183. http://dx.doi.org/10.1023/A:1006423220775

Rodríguez-Domínguez, L., García-Sánchez, I., \& Gallego-Álvarez, I. (2009). Codes of Ethics in Spanish Corporations: An exploratory content analysis. International Journal of Law and Management, 51, 291-309. http://dx.doi.org/10.1108/17542430910988892

Ruiz-Lozano, M, \& Araque-Padilla, R. (2013). ¿Son los Códigos Éticos un Instrumento para Proteger al Stakeholder Consumidor? Un estudio de los sectores financiero, energético y de telefonía españoles. Spanish Accounting Review, 16(2), 159-168. http://dx.doi.org/10.1016/j.rcsar.2013.07.005

Russo, A., \& Perrini, F. (2010). Investigating Stakeholder Theory and Social Capital: CSR in Large Firms and SMEs. Journal of Business Ethics, 91, 207-221. http://dx.doi.org/10.1007/s10551-009-0079-z

Schwartz, M. (2001). The Nature of the Relationship Between Corporate Codes of Ethics and Behavior. Journal of Business Ethics, 32, 247-262. http://dx.doi.org/10.1023/A:1010787607771

Sethi, P. (2003). Setting Global Standard: Guidelines for Creating Codes of Conduct in Multinational Corporations. Hoboken, NJ: J. Wiley.

Singh, J. (2006). A Comparison of the contents of the Codes of Ethics of Canada's Largest Corporations in 1992 and 2003. Journal of Business Ethics, 64, 17-29. http://dx.doi.org/10.1007/s10551-0052408-1

Singh, J. (2011). Determinants of the Effectiveness of Corporate Codes of Ethics: An Empirical Study. Journal of Business Ethics, 101, 385-395. http://dx.doi.org/10.1007/s10551-010-0727-3 
Szabo, A. (2008). The corporate social responsibility: An opportunity for SMEs. Available online at:: http://www.unglobalcompact.org/http://www.unece.org/indust/sme/ResponsibleEntrepreneurship.

Toro, D. (2006). El enfoque estratégico de la responsabilidad social corporativa: Revisión de la literatura económica. Intangible Capital, 14(2), 338-358.

Turyakira, P., Venter, E., \& Smit, E. (2012). Corporate Social Responsibility for SMEs: A proposed hypothesised model. African Journal of Business Ethics, 6, 106-118. http://dx.doi.org/10.4103/18177417.111015

Valentine, S., \& Barnett, T. (2003). Ethics Code Awareness, Perceived Ethical Values, and Organizational Commitment. Journal of Personal Selling and Sales Management, 23, 359-367.

Winkler, I. (2011). The Representation of Social Actor in Corporate Codes of Ethics. How Code Language Positions Internal Actors. Journal of Business Ethics, 101, 653-665. http://dx.doi.org/10.1007/s10551-011-0762-8

Wood, G., Svensson, G., Singh, J., Carasco, E., \& Callaghan, M. (2004). Implementing the Ethos of Corporate Codes of Ethics: Australia, Canada and Sweden. Business Ethics: A European Review, 13: 389-403. http://dx.doi.org/10.1111/j.1467-8608.2004.00378.x

Intangible Capital, 2016 (www.intangiblecapital.org)

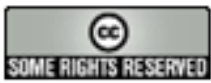

Article's contents are provided on an Attribution-Non Commercial 3.0 Creative commons license. Readers are allowed to copy, distribute and communicate article's contents, provided the author's and Intangible Capital's names are included. It must not be used for commercial purposes. To see the complete license contents, please visit http://creativecommons.org/licenses/by-nc/3.0/. 